பராபரக்கண்ணியில் தாயுமானவர், குணங்குடியாரின் இறையுணர்வு

ப. சசிரேகா அ, •

அ தமிழ்த்துறை, குரு நானக் கல்லூரி (தன்னாட்சி), சென்னைப் பல்கலைக்கழகம், சென்னை-600042, தமிழ்நாடு, இந்தியா

\title{
The Spiritual Expressions of Dhayumaanavar and Gunangudiyar in
}

\section{Paraparakkanni}

\section{P. Sasireka ${ }^{\text {a, }}{ }^{*}$}

a Tamil Department, Guru Nanak College, Madras University, Chennai-600042, Tamil Nadu, India.

* Corresponding Author: sasirekapalani@gmail.com

Received: 04-01-2021 Revised: 12-04-2021

Accepted: 19-04-2021

Published: $30-04-2021$

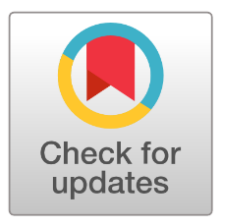

\begin{abstract}
Spiritual literature takes the foremost role in the history of literature. Spiritual literature has the tendency to impact our spirit and leads us afloat on the ocean of happiness. Every religion has been at certain points of time propounded by a teacher and later spread throughout the globe by their students. However some of the worship methods predate the era of literature. Islam is a religion that grew through the teachings of Nabi. Many saints have expressed the immense state of spiritual attainment by forsaking the material joys of the world through their songs. Many religions have used the Tamil language as a medium of expressing their ideology. All religions are unified by their emotive spirituality. Dhayumanavar and Gunangudiyar occupy a significant position in the history of literature. Eventhough their means to spirituality differs they express similar spiritual experiences. The purpose of this article is to research on the similarity observed in their mode of prayer and descriptions in each of their Paraparakkanni.
\end{abstract}

Keywords: Paraparakanni, Spiritual expression, Beginning and end, Globe, Earth.

\section{முன்னுரை}

சமயங்கள் ஒவ்வொன்றும் ஒவ்வொரு காலத்தில், ஒவ்வொரு ஆசிரியரால் நிறுவப்பட்டுப் பின் அவர்தம் மாணவர்களால் உலகில் பரப்பப்பட்டுள்ளது. ஆனால் சைவசமயத்தில் சிவவழிபாடு வரலாற்றுக் காலத்துக்கு முற்பட்டது. இஸ்லாம் சமய வழிபாடு நபிகள் நாயகத்தின் வழி வளர்ந்துள்ளது. உலகவாழ்வின் இன்பங்களில் உள்ள பற்றை விடுத்து பக்திப்பரவசம் மேலோங்க ஆனந்த நிலையை எய்திய ஞானிகளில் பலர் தங்களது இறையனுபவத்தைப் பாடல்கள் வாயிலாக வெளிப்படுத்தினர். அப்பாடல்கள் நமக்கு இறையனுபவத்தை வழங்குகின்றன. தாயுமானவரும், குணங்குடியாரும் இறைவனை வழிபாடு செய்தவிதம் வேறுவேறாக இருந்தாலும், இருவரும் ஒரேமாதிரியான இறையனுபவத்தையே வெளிப்படுத்துகின்றனர். இறைவனை வழிபாடு செய்யும் விதமும், வர்ணிக்கும் விதமும் ஒன்றாகவே அவர்தம் பராபரக்கண்ணியில் இடம்பெற்றுள்ளது.

\section{இறைவன் எங்கும் நிறைந்தவன்}

இயற்கை என்பது இறைவனால் உருவாக்கப்பட்டது. இயற்கையை முழுமையாக அறிந்தவனும், அதனை தன்னுடைய கட்டுப்பாட்டில் வைத்திருப்பவனும் இறைவன்தான். இதனை உணர்ந்த 
தாயுமானவரும், குணங்குடியாரும் இறைவுணர்வை ஒரே தன்மையில் வெளிப்படுத்தியுள்ளனர். இருவரின் சமயங்கள் வேறுவேறாக இருந்தாலும், இவ்விருவரும் இறைவன் அண்டம், உலகம் என்று எல்லா இடத்தும் நிறைந்தும், எல்லாவற்றிற்கும் ஆதியாகவும், எல்லாமாகவும் இ இருப்பதாகக் கூறுகின்றனர்.

“அண்டம் அனைத்திலுமாய் அப்பாலுக்கு அப்பாலும்

கொண்டநின்னை யார்அறிந்து கொள்வார் பராபரமே” (தா. ப.பா : 114)

“ஆதிஅந்தம் நீகுருவாய் ஆண்டதுஅல்லால் நின்னைஅன்றிப்

போதனையும் உண்டோ புகலாய் பராபரமே”

(தா. ப.பா : 240)

“அண்ட புவனமென்று மாடுதிருக் கூத்தினையான்

கண்டு மகிழ்ந்திடவே காட்டாய் பராபரமே"

(கு.ப.பா : 1)

“ஆதியா யாண்டவனாய் அஃததுவாய் நின்றபெருஞ்

சோதியாய் நின்மலமாய்ச் சூழ்ந்தாய் பராபரமே” (கு.ப.பா : 2)

அண்டம் என்பது அனைத்து கோள்களையும் உள்ளடக்கிய ஒன்றாகும். இறைவன் அண்டத்திற்கு அப்பாலும், எல்லா இடத்தும் நிறைந்து இருப்பதாகவும், இறைவனை அறிந்து கொள்வது கடினமான செயலாகவும் வெளிப்படுத்துகின்றனர்.

\section{எத்திசையும் இறைவனைத் தேடுதல்}

ஐம்புலன்களுக்கும் அப்பாற்பட்ட நிலைதான் இறைவன். புலன்களால் அறியப்படுவது உண்மையாகத் தோன்றினாலும், சில சமயங்களில் பொய்யானதாக மாறிவிடுகின்றது. தாயுமானவரும், குணங்குடியாரும் இறைவனை பல இடங்களில் தேடியும், அவர்களால் இறைவனைக் காணமுடியவில்லை என்பதை,

‘தேடினேன் திக்குஅனைத்தும் தெண்டனிட்டேன் சிந்தைநைந்து

வாடினேன் என்மயக்கம் மாற்றாய் பராபரமே"

(தா. ப.பா : 200)

“செகமுழுதுஞ் சுற்றியுமுன் சீர்பாதங் காணாமற்

றிகைத்தழுதே னீயுந் தெரிவாய் பராபரமே” (கு.ப.பா : 77)

என்கின்றார். இறைவன் என்பவன் எல்லா திசைகளிலும் நிறைந்து இருக்கின்றான். இறைவனை உணர்வாக அறிபவர்கள் மதங்களுக்கு அப்பாற்பட்டவர்கள். அவ்விடத்தில் அன்பும், கருணையும்தான் இருக்கும். எங்கு தேினாலும் இறைவனைக் காணமுடியவில்லையே என்று வருத்தப்படுகின்ற உணர்வும், இருவருக்கும் ஒன்றாகவே காணப்படுகின்றது.

\section{இறைவனை வர்ணிக்கும் விதம்}

சிலர் இறைவனை தாயாகவும், தந்தையாகவும், காதலியாகவும், பிள்ளையாகவும் பாவித்து பாடியுள்ளனர். பாற்கடலை கடையும்பொழுது கடலிலிருந்து அமுதம் கிடைத்தது. அதனால்தான் பெரும்பாலானோர் கடல் என்றாலே அமுதம் என்கின்றனர். இவர்களோ இறைவனை அமுதமாகவும், நவரசமாகவும், கரும்பிலிருந்து கிடைக்கும் பாகாகவும், கடலாகவும், கருப்புவட்டாகவும், கற்கண்டாகவும், கண்ணாகவும், முத்தாகவும், பவளமாகவும் வர்ணிக்கின்றனர்.

“கடல்அமுதே தேனேஎன் கண்ணே கவலைப்

படமுடியாது என்னைமுகம் பார்நீ பராபரமே"

(தா. ப.பா : 32) 
“கன்னல்தரும் பாகாய்க் கருப்புவட்டாய்க் கற்கண்டாய்

இன்னமுதாய் என்உள் இருந்தாய் பராபரமே"

(தா. ப.பா : 248)

“தேடக் கிடையாத திரவியமே தேன்கடலே

ஈடுனக்கு முண்டோ விறையே பராபரமே"

(கு.ப.பா : 15)

“கரும்பே நவரசமே கடலமுதே கண்மணிக்குள்

அரும்பொருளாய் நின்ற வழகே பராபரமே"

(கு.ப.பா : 41)

தாயுமானவரை அடியொற்றி பாடல்களைப்பாடிய குணங்குடியாரின் பாடல்கள் இஸ்லாமியத்தின் பெருமையை வெளிப்படுத்துவதோடு தாயுமானவரின் கருத்துகளையே பிரதிபலிக்கின்றது. உயிரினங்கள் தங்கள் குழந்தைகளைக் கண்ணுக்குள் வைத்து காப்பது இயல்பு. கண்கள் வழியாகத்தான் இறைவனைக் காணமுடியும்.

“கண்ணே கருத்தேஎன் கற்பகமே கண்நிறைந்த

விண்ணே ஆனந்தவியப்பே பராபரமே"

(தா. ப.பா : 10)

“கண்ணே கருத்தேயென் கண்மணியே கண்ணிறைந்த

விண்ணடங்கா வெட்ட வெளியே பராபரமே” (கு.ப.பா : 50)

இவர்களோ இ இறைவைக் கண்ணாகவும், கண்ணில் உள்ள மணியாகவும், கண்ணில் கலந்திருக்கும் அருளாகவும் எண்ணி இன்பம் அடைகின்றனர்.

\section{இரங்கி அருள் தாராய்!}

இருவரும் துறவு வாழ்க்கையை மேற்கொண்டவர்கள். இறறவன்மீது பற்றுடையவர்கள். இவர்களுக்கு இறைவன் இன்னும் காட்சிதரவில்லை. அதனால் இவர்கள் இறைவனிடம் இரங்கி அருள் புரியவேண்டும் என்று வேண்டுகின்றனர்.

“கன்றினுக்குச் சேதா கனிந்துஇரங்கல் போலஎனக்கு

என்றுஇரங்கு வாய்கருணை எந்தாய் பராபரமே” (தா.ப.பா : 34)

“எவ்வுயிரும் என்உயிர்போல் எண்ணி இரங்கவும்நின்

தெய்வ அருள்கருணை செய்யாய் பராபரமே"

(தா. ப.பா : 65)

"ஆனாலுமே ழையடியேனுக் காதரவாயத்;

தானாயிரங்கி யருள்டாராய் பராபரமே"

(கு.ப.பா : 48)

“ஆனாலு முன்பாதம் யாசித் திருப்பதற்குத்

தானா யிரங்கி யருள்டாராய் பராபரமே” (கு.ப.பா : 8)

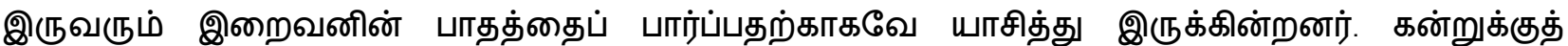
தாய்பசு கனிந்து இரக்கப்படுவதுபோல இறைவனை இரங்கும் படியும், எல்லா உயிரையும் தன் உயிர்போல் எண்ணுவதற்கு அருள்புரிய வேண்டும் என்றும் வேண்டுகின்றனர்.

\section{உறவினர் உறவும் இறைவனின் உறவும்}

உறவினர்களின் வேண்டுதலுக்கு இணங்கி தாயுமானவர் திருமணம் புரிந்து ஆண்குழந்தையைப் பெற்றெடுத்துபின் இறைவன் மீது உள்ள பற்றால் துறவு வாழ்க்கையை மேற்கொண்டார். குணங்குடியாரோ திருமணம் நடக்கும் பொழுது, திருமணத்தை நிறுத்திவிட்டு இறைவன் மீது 
பற்றுகொண்டு துறவு வாழ்க்கையை மேற்கொண்டார். இவர்கள் இருவரும் இறைவன்தான் தனக்கான சிறந்த உறவு என்ற கொள்கையோடு இருந்தனர். இதனால் மற்ற உறவுகளை வெறுத்தனர். துறவு வாழ்க்கைக்கு மற்ற உறவுகளால் எந்த பயனுமே இல்லை என்று கருதினர்.

“தாய்இருந்தும் பிள்ளை தளர்ந்தாற்போல் எவ்விடத்தும்

நீஇருந்தும் நான்தளர்ந்து நின்றேன் பராபரமே” (தா. ப.பா : 349)

“பேராற் பெரிய பெரும்பொருளே பேதைதனக்

காரா ரிருந்தும் பலனாமோ பராபரமே"

(கு.ப.பா :6)

தாய் இருந்தால் பிள்ளையை அழுவதற்கு விடமாட்டாள். எனக்கு தாய்போல நீ இருந்தும் ஏன் என்னை அழவைக்கின்றாய் என்று இறைவனோடு உரிமையோடு கேட்கின்றனர். மேலும் சாதிசனத்துடன் இருந்தால் துன்பமே நேர்கின்றது. தாய் உயிரோடு இருக்கும்போது பிள்ளை இறப்பதைப்போல், நீ இருக்கும்போதே நான் வாடி கலங்குகிறேன். இதனால் உறவே வேண்டாம் என்கின்றனர்.

\section{வேதத்தின்வழி இறைவனை அடையலாமா?}

மதம் மக்களை நல்வழிப்படுத்துவற்காக உருவானது. கடவுள் மீீது கொண்டிருக்கும் பக்தி, நம்வாழ்க்கையை நல்வழியில் செலுத்துவதற்கு உதவுகின்றது. முக்திபெறுதல் என்பது இறைவனின் பாதத்தை அடைவதற்கான வழியாகும். இது மீண்டும் பிறத்தல் இறத்தல் இல்லாத நிலையாகும்.

“வேதாந்தம் சித்தாந்தம் வேறுஎன்னார் கண்களிக்கும்

நாதாந்த மோன நலமே பராபரமே”

(தா. ப.பா : 206)

“வேதமறைப் பொருளை வேதாந்தத் துட்கருவை

ஓதியுனை யறிந்தாருண்டோ பராபரமே"

(கு. ப.பா : 3)

“வேஷந் தனைப்போட்டு வேதாந்த சாத்திரத்தைக்

காசுபணம் பறிக்கக் கற்றேன் பராபரமே"

(கு.ப.பா : 96)

உள்ளத்தில் எந்தவிதமான தீய எண்ணமும் இ இருக்கக்கூடாது. வேதத்தைக் கற்றால் இறைவனைக்காண முடியும் என்று அனைவரும் நம்புகின்றனர். ஒருவர் முக்திபெற வேண்டுமானால் இறைவனின் மந்திரத்தை ஓதவேண்டும். வேதாந்த சாத்திரத்தைக் காசுபணம் சம்பாதிப்பதற்காகவே கற்றேன் என்றும், வேதாந்தத்தை ஓதி இறைவனைப் பார்த்தவர்கள் எவரேனும் இருக்கின்றனரா என்றும் கேட்கின்றனர். இறைவன் பல சமயமாகவும், பல உருவமாகவும் இருக்கின்றான்.

"ஒன்றே பலவே உருவே அருவேயோ

என்றே அழைப்பது உன்னைஎன்றோ பராபரமே” (தா. ப.பா : 118)

"பார்க்கப் பலவிதமாய் பலசமய மாகவொரு

வாக்கை வெளிப்படுத்தி வைப்பாய் பராபரமே”

(கு. ப.பா : 65)

“தானம் தவம்தருமம் சந்ததமும் செய்வர்சிவ

ஞானம் தனை அணையநல்லோர் பராபரமே"

(தா.ப.பா : 158)

“மந்திரத்துக் கெட்டா மறைப்பொருளே மன்னுயிரே

சேர்ந்தவெழு தோற்றத்தின் சித்தே பராபரமே” (கு.ப.பா : 11) 
இறைவன் என்பவன் பார்ப்பதற்கு பல வகையான உருவத்தோடும், பல சமயமாகவும் இருப்பவன். மந்திரத்திற்கு அப்பாற்பட்டவன்தான் இறைவன். அதனால் தானம், தருமம் போன்ற அறச்செயல்களைச் செய்தால் மட்டுமே அடையமுடியும் என்று இருவரும் கூறுகின்றனர்.

\section{முடிவுரை}

தாயுமானவரும், குணங்குடியாரும் இறைவன் என்பவன் அண்டம் முழுவதும் நிறைந்தவன் என்றும், அமுதம் போன்றவன் என்றும் இருவரும் ஒரேவிதமாகவே வர்ணித்துள்ளதைக் காணமுடிகின்றது. உறவைவிட்டு விலகி, துறவு வாழ்க்கை மேற்கொள்வதன் மூலமும், தானம், தருமம் செய்வதன் மூலமும் இறைவனை அடையமுடியும் என்று கூறுவதைக் காணமுடிகிறது. இருவருடைய பராபரக்கண்ணியிலும், ஒத்தக்கருத்துகளே நிலவுகின்றன. இருவருடைய சமயத்தை நீக்கிவிட்டு பார்த்தால் இறைவன் என்பவன் ஒருவனே. ஒவ்வொருவரும் ஒவ்வொருஉருவத்தில் இறைவனைக் காண்கின்றனர் என்பது புலப்படுகின்றது. இறைவன் என்பவன் பல உருவத்தோடும், உருவம் இல்லாமலும் இருக்கின்றான் என்பதை இருவருடைய பராபரக்கண்ணியிலும் அறியமுடிகின்றது. இறைநம்பிக்கை இன்று பல்வேறு பரிமாணங்களைப்பெற்று, அதன் உண்மை நிலையில் இருந்து மாறிவிட்டது. ஆனால் இறையுணர்வு என்பது மதம் கடந்த ஒன்றாகவே தாயுமானவரும், குணங்குடியாரும் தங்களுடைய பராபரக்கண்ணியில் வெளிப்படுத்தியுள்ளனர்.

\section{References}

Meiyappan, S., (2002), Thayumaanavarkal Bhatalkal , Mani Vaasakar Publication, Chennai, India.

Gunangudi Musdhaan Shakibu Avarkal Bhadalkal (March-1985), The South India Saiva Siddhantha Publishing Society, Chennai, India.

Funding: NIL

Acknowledgement: NIL

Conflict of Interest: NIL

About the License:

\section{(c) (1)}

Attribution 4.0 International (CC BY 4.0)
(C) The author 2021. The text of this article is licensed under a Creative Commons Attribution 4.0 International License 